\title{
Erratum: Topological Optical Waveguiding in Silicon and the Transition between Topological and Trivial Defect States [Phys. Rev. Lett. 116, 163901 (2016)]
}

\author{
Andrea Blanco-Redondo, Imanol Andonegui, Matthew J. Collins, Gal Harari, Yaakov Lumer, \\ Mikael C. Rechtsman, Benjamin J. Eggleton, and Mordechai Segev \\ (Received 3 August 2016; published 13 September 2016)
}

DOI: 10.1103/PhysRevLett.117.129901

In the associated Letter, we have omitted a precise description of the nature of the topological protection associated with the interface state we study, which is potentially misleading to the reader.

To rectify this, we would replace the following statement (left-hand column, page 4): "The experimental measurements and the simulations follow the same trends, although the quantitative agreement between them is not as good as in the structures with a topological defect. This is due to the fact that the fabrication imperfections significantly affect the optical transport in the extended structure experiencing discrete diffraction, whereas the topologically protected defect state propagates immune to them" with this one: "The fact that the wave function amplitude remains near-zero on every other waveguide - a consequence of the topological nature of the state in the SSH model—is an indication that we have excited this state almost exclusively. Indeed, the introduction of off-site disorder (i.e., randomizing the positions of the waveguides) would not change this property."

Furthermore, we would replace the following statement (right-hand column, page 4): "Finally, Fig. 5(c) demonstrates that the topological defect mode maintains its power distribution at the output of the structure across the entire wavelength range" with this one: "Finally, Fig. 5(c) demonstrates that the optical power distribution remains near zero on alternating waveguides, across the entire wavelength range, a signature of the topological nature of the state."

We further note that the vertical axes in Figs. 2(a) and 2(b) should be labeled $k_{z}$, rather than $k_{x}$. The same replacement should be made in the caption of Fig. 2 as well. 